\title{
A Broadband Dual-Polarized Antenna for LTE Base Stations Applications
}

\author{
Qiuqiang Luo \\ College of \\ Communication \\ Engineering \\ Chengdu University \\ Information of \\ Technology \\ Chengdu, China
}

\author{
Meng Li \\ College of \\ Communication \\ Engineering \\ Chengdu University \\ Information of \\ Technology \\ Chengdu, China
}

\author{
Jiaming Xia \\ College of \\ Communication \\ Engineering \\ Chengdu University \\ Information of \\ Technology \\ Chengdu, China
}

\author{
Hao Zou \\ College of \\ Communication \\ Engineering \\ Chengdu University \\ Information of \\ Technology \\ Chengdu, China
}

\begin{abstract}
In this paper, A broadband Dual-polarization base station antenna has been presented for LTE mobile communication system application. The antenna adopts metal matrix die casting technology, and the model structure has good machining consistency and engineering practicability. Meanwhile, the compensation performance can be provided by attaching parasitic branches at the end of the radial arm. The simulated results demonstrate the proposed antenna can operate at the frequency range of LTE $(1.68-2.69 \mathrm{GHz})$ band with the voltage standing wave ratio is less than 1.5 and the isolation is more than $22 \mathrm{~dB}$. The developed antenna element achieves average gains of $9.822 \mathrm{dBi}$ and a half power beam width of $63 \pm 5^{\circ}$. It suitable for LTE mobile communication base station applications.
\end{abstract}

Keywords: dual-polarization; base-station antenna; broadband; LTE mobile communication

\section{INTRODUCTION}

In the design of base station antenna, broadband antenna, especially $\pm 45^{\circ}$ dual-polarize antenna that can improve channel capacity and combat multipath interference, have been widely studied. Currently, two production processes are commonly used to make base station antennas. One is to design the antenna by. the PCB printing process [1-5] with have an advantage of low profile, low cost and easy of mass fabrication [6], but its stability and service are limited. The other is a metal finale process with long life and high stability, which is widely used in the market, but its production cost is relatively high. Literature [7] and [8] have a similar model structure, and a downward-derived parasitic stub is added at the end of the radiating element arm to expand the antenna operating band and increase the antenna gain value. However, the hollow design of the radiating element arm puts forward higher requirements on the machining accuracy, particularly the right-angled parasitic branch loaded in the middle of the radiation arm of literature [7], which has poor processing consistency and stability.

In this paper, a new base station antenna radiating element is proposed, and the designed model has better mechanical stability. By loading a parasitic stub with a certain physical length at the end of the radiation arm, the antenna operating band can be effectively expanded, and at the same time, the antenna can be compensated for certain performance and has better flexibility. The proposed antenna radiating element has a stable structure, and the operating frequency band can cover 1.68-2.69 GHz, has good circuit performance and radiation performance over the entire operating frequency, and has high engineering application value. It can be a candidate for LTE mobile communication base station applications.

\section{ANTENNA DESIGN}

The configuration of the proposed antenna is shown in Figure 1. The antenna radiating element model structure comprises a radiating arm, a coaxial cable and a reflective bottom plate, and two radiating arms on the diagonal line form a polarize radiating element, and mutually orthogonally form a $\pm 45^{\circ}$ dual polarized antenna. The antenna radiating arm adopts a rectangular and circular trapezoidal hollow structure design, which increases the current of the radiating arm through the route, reduces the size of the antenna, and has better mechanical stability. The end of the radiating arm is open for easy installation of parasitic stubs. The balun has the function of providing balance feeding and supporting radiating arm and adopts a faux pyramid structure design, which has better processing and pouring consistency. The reflective bottom plate is designed with a stepped structure and is stamped from aluminum alloy material.

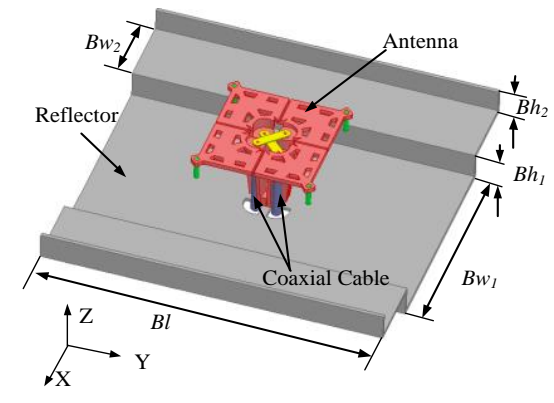

(a) 


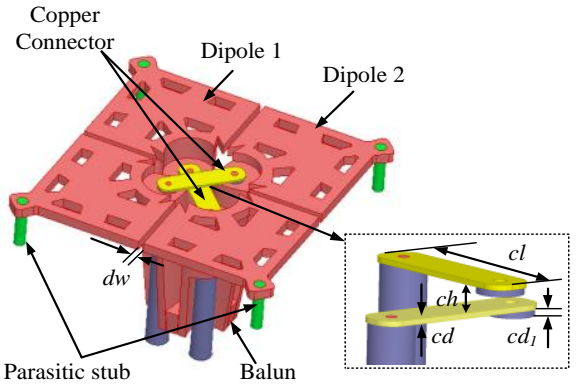

(b)

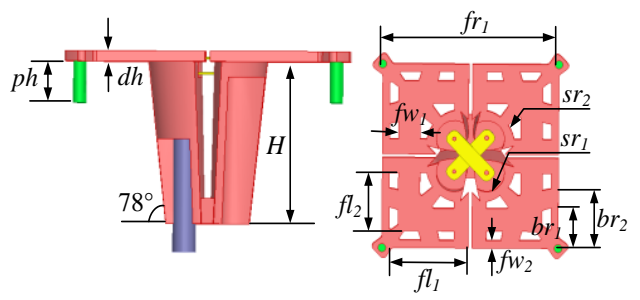

(c)

Figure1. Configuration of the proposed antenna. (a) overall diagram of the antenna and reflector, (b) 3D view, and (c) side view and bottom view.

The antenna is analyzed and optimized by high-frequency electromagnetic simulation software Ansoft HFSS15, and the parameter values defined in Figure. 1 are shown in table 1

Table 1. Parameter value for the antenna (units:mm)

\begin{tabular}{|c|c|c|c|}
\hline parameter & value & parameter & value \\
\hline$B l$ & 120 & $c l$ & 16.7 \\
\hline$B w_{1}$ & 80 & $c d_{1}$ & 0.8 \\
\hline$B w_{2}$ & 30 & $\mathrm{H}$ & 32 \\
\hline$B h_{1}$ & 15 & $d h$ & 2 \\
\hline$B h_{2}$ & 12 & $p h$ & 8 \\
\hline$d w$ & 1 & $f l_{1}$ & 24 \\
\hline$c d$ & 0.4 & $f l_{2}$ & 16.2 \\
\hline$c h$ & 2.2 & $s r_{1}$ & 2.02 \\
\hline$f w_{1}$ & 6.2 & $s r_{2}$ & 5 \\
\hline$f w_{2}$ & 2 & $b r_{1}$ & 11 \\
\hline$f r_{1}$ & 49 & $b r_{2}$ & 16 \\
\hline
\end{tabular}

\section{ANALYSIS OF SIMULATION RESULT}

The proposed antenna operating band can be effectively extended by loading parasitic stubs. The simulation results of the antenna voltage standing wave ratio (VSWR) with/without parasitic stub are shown in the Figure. 2(a), it can be see that the operating frequency band with the VSWR is less than 1.5 in the low-frequency band is extended downward by $70 \mathrm{MHz}$.

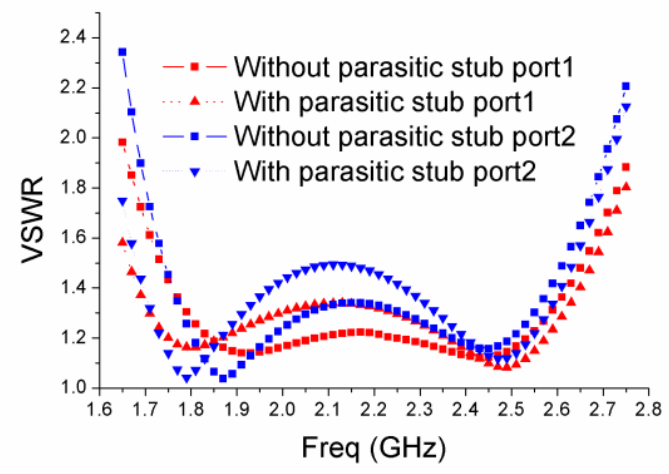

(a)

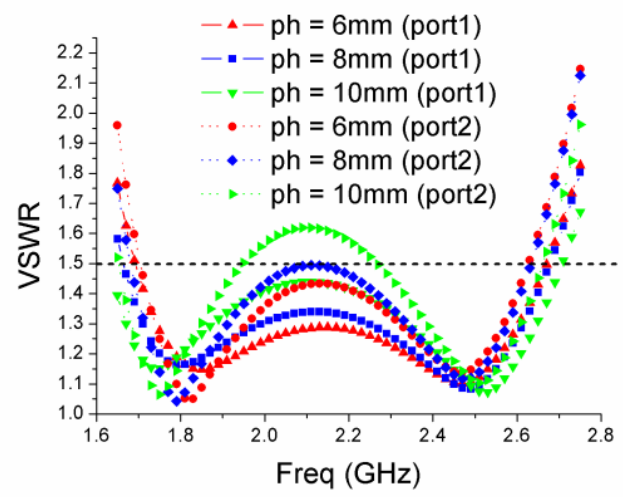

(b)

Figure. 2 Simulated VSWRs of the proposed antenna. (a) with/without parasitic stub, (b) with different $\mathrm{ph}$.

The physical length ph of the parasitic stub is the main parameter affecting the performance of the circuit in the operating frequency band of the antenna. As shown in Figure. 2(b), the VSWR curve in the antenna band is simulated respectively at $\mathrm{ph}=6,8,10 \mathrm{~mm}$. It can be seen that the $\mathrm{ph}=8 \mathrm{~mm}$ comprehensive performance is better under the performance requirement that the VSWR less than 1.5.

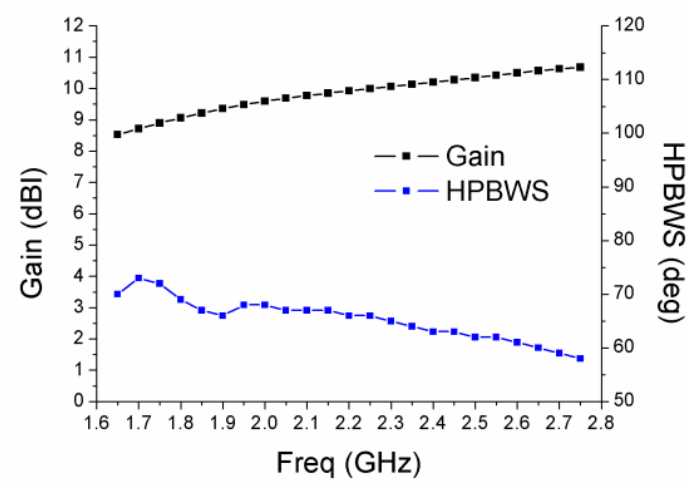

(a) 


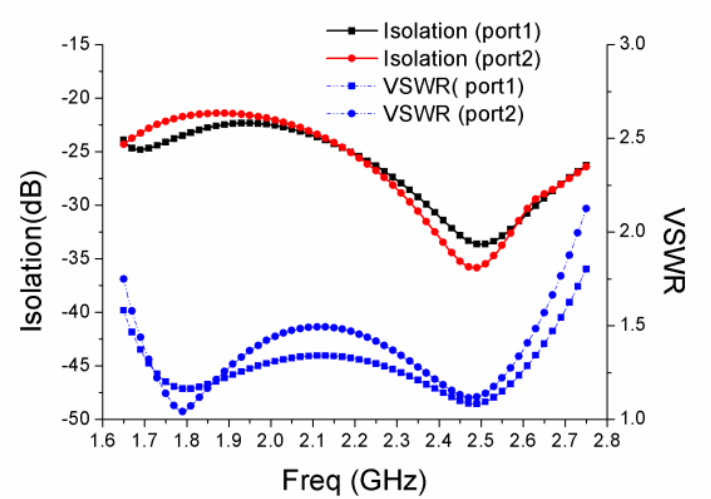

(b)

Figure 3. Simulated results of the proposed antenna (a) gain and HPBWS, (b) Isolation and VSWR

The antenna isolation and VSWR simulation results are shown in Figure. 3(a), the antenna isolation is greater than $22 \mathrm{~dB}$, the operating frequency band is $1.68-2.69 \mathrm{GHz}(\mathrm{VSWR}<1.5)$, and the antenna gain value and the half power beam width (HPBWS) simulation result are obtained. As shown in Figure. 3 (b), the average gain is $9.822 \mathrm{dBi}$ and the half power beam width is $63 \pm 5^{\circ}$

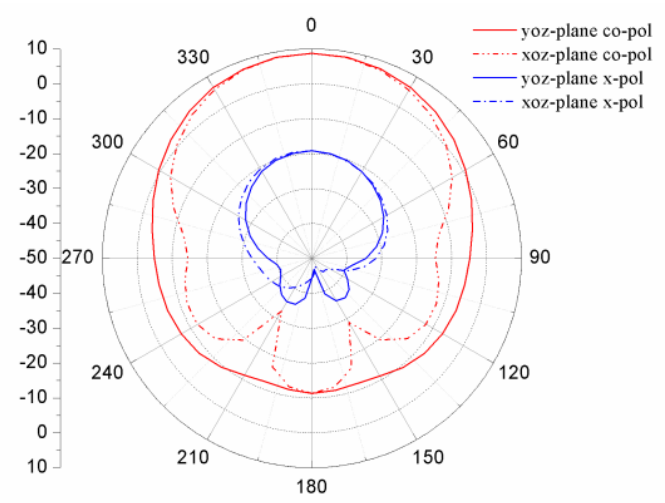

(a)

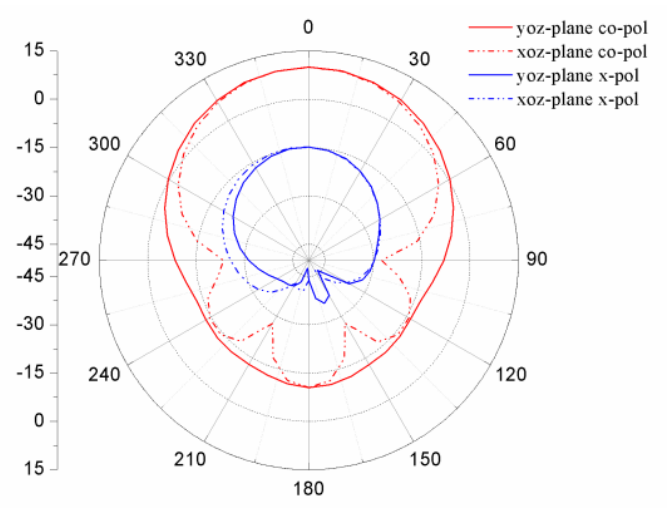

(b)

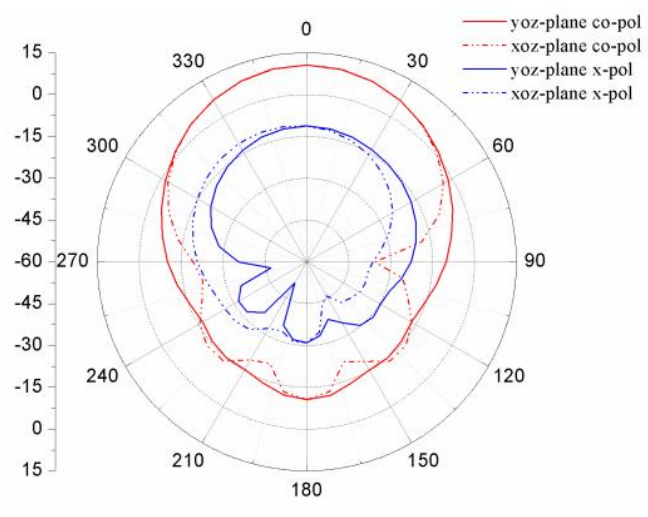

(c)

Figure 4. Radiation patterns of the proposed antenna (a) $1.7 \mathrm{GHz}$, (b) $2.2 \mathrm{GHz}$, and (c) $2.7 \mathrm{GHz}$.

1.7, 2.2, $2.7 \mathrm{GHz}$ main polarization and cross polarization, xozplane and yoz-plane antenna pattern simulation results are shown in Figure. 4, the results show that the antenna has a stable radiation pattern, discrimination rate is $21.89 \mathrm{~dB}\left(\theta=0^{\circ}\right)$, and $12.11 \mathrm{~dB}\left(\theta= \pm 60^{\circ}\right)$, which satisfies the base station antenna design requirements.

\section{CONCLUSION}

In this paper, a metal base station antenna radiating element is designed. The antenna operating frequency band is extended by loading the detachable parasitic stub. As a result, the operation band of the antenna for VSWR $<1.5$ is about $46.22 \%$ (1.68$2.69 \mathrm{GHz}$ ), stable radiation patterns with an average gain of $9.822 \mathrm{dBi}$ and a half power beam width $63 \pm 5^{\circ}$ at the operating frequency are obtained. The structure has good mechanical stability and can be for base station systems applications.

\section{REFERENCES}

[1] Y. H. Cui, R. L. Li, and P. Wang, "A Novel Broadband Planar Antenna for 2G/3G/LTE Base Stations," IEEE transaction on antennas and propagation, vol. 61, no. 5,page: 2767-2773, May. 2013.

[2] C. H.Wang, Y. K. Chen, and S. W. Yang, "Dual-band DualPolarized Antenna Array With Flat-Top and Sharp Cutoff Radiation Patterns for 2G/3G/LTE Cellular bands," IEEE transaction on antennas and propagation, vol. 66, no. 11, page: 5907-5916, Nov. 2018.

[3] D. Z. Zhang, Q. X. Chu, "A Dual-Polarized Planar Antenna Using Four Folded Dipoles and Its Array for Base Stations," IEEE transaction on antennas and propagation, vol. 64, no. 12, page: 5536-5542, Dec. 2016.

[4] Y. H. Cui, R. L. Li, and H. Z. Fu, "A Broadband DualPolarize Planar Antenna for 2G/3G/LTE Base Station," IEEE transaction on antennas and propagation, vol. 62, no. 9, page: 4836-4840, Sept. 2014.

[5] H.Huang, Y. Liu, S. X. Gong, “A Broadband Dual-Polarize Base Station Antenna With Sturdy Construction," IEEE antenna and wireless propagation letters, vol. 16, page: 665-668, 2017.

[6] Q. Y. Zhang, Y. Gao, "A Compact Broadband DualPolarized Antenna Array for Base Station," IEEE antenna and wireless propagation letters, vol. 17, no. 6,page: 1073 1076, June . 2018.

[7] Y. J. He, W. Tian, and L. Zhang, “A Novel Dual-Polarized 
International Journal of Computer Applications Technology and Research

Volume 8-Issue 07, 272-275, 2019, ISSN:-2319-8656

Electrical Downtilt Base Station Antenna for 2G/3G Applications," IEEE Access, vol 5, page: 15241-15249, 2017.

[8] Y. J. He, J. Li, S. W. Wong, et al, "A Miniaturized Base Station Antenna With Novel Phase Shifter for 3G/LTE Applications," IEEE Access, vol. 6, page: 52877-52885, 2018. 